\title{
Ultrastructure of the Pathogenic Bacteria Mobiluncus mulieris
}

\author{
Jennifer Costello ${ }^{1}$, Jonathan Blaize ${ }^{1}$, William J. L’Amoreaux ${ }^{1,2}$ and Elena C. McCoy ${ }^{1}$ \\ ${ }^{1}$ Department of Biology, CUNY College of Staten Island, 2800 Victory Blvd., Staten Island, NY \\ 10314 \\ ${ }^{2}$ Advanced Imaging Facility of the College of Staten Island, 2800 Victory Blvd., Staten Island, NY \\ 10314
}

Mobiluncus spp. are gram variable anaerobic rods found associated with bacterial vaginosis. Recent molecular analyses of Falcivibrio spp. has identified these species as misclassified Mobiluncus [1]. We therefore will use Mobiluncus for further description of these organisms.

Clinical isolates of M. mulieris (ATCC) were maintained on Columbia CNA Blood Agar under anaerobic conditions [2]. For growth of cultures for electron microscopic analyses, cells were grown anaerobically in thioglycolate broth for $24 \mathrm{hr}$ at $37^{\circ} \mathrm{C}$, collected by centrifugation and transferred immediately to $4 \%$ paraformaldehyde, $2.5 \%$ glutaraldehyde in either sodium cacodylate or Sorenson's phosphate buffer. Cells were fixed for $24 \mathrm{hr}$, rinsed in appropriate buffer, and post-fixed in $1 \% \mathrm{OsO}_{4}$. The cells were dehydrated in an ascending ethanol series and transferred to propylene oxide as a transition fluid. The cells were then infiltrated in Epon-Araldite (substituted; Electron Microscopy Sciences) and embedded in BEEM capsules. The resin was cured $24 \mathrm{hr}$ at $60^{\circ} \mathrm{C}$, semithick sections were obtained (Leica Ultracut UCT) for light microscopic analysis. Thin sections were obtained using a Diatome diamond knife, picked up on copper grids, stained in uranyl acetate and a modified Sato's lead stain [3]. Sections were imaged with a Philips CM100 and digital images collected (SIA-7C CCD camera; Scientific Instruments and Applications, Inc.; Duluth GA).

Light microscopic analyses revealed variable staining rods, some with darkly stained inclusions in the cytoplasm (Figure 1). Examination by transmission electron microscopy revealed several electron translucent bodies (Figure 2) as well as filamentous processes (Figure 3). With higher resolution, these processes appear to be pili (Figures $4 \& 5$ ). These preliminary results will be used to further characterize the inclusion and filamentous processes.

[1] L. Hoyles, et al., Syst Appl Microbiol, 27 (2004) p. 72.

[2] H. J. Smith and H. B. Moore, J Clin Microbiol, 26 (1988) p. 1134.

[3] T. Hanaichi, et al., J Electron Microsc (Tokyo), 35 (1986) p. 304. 


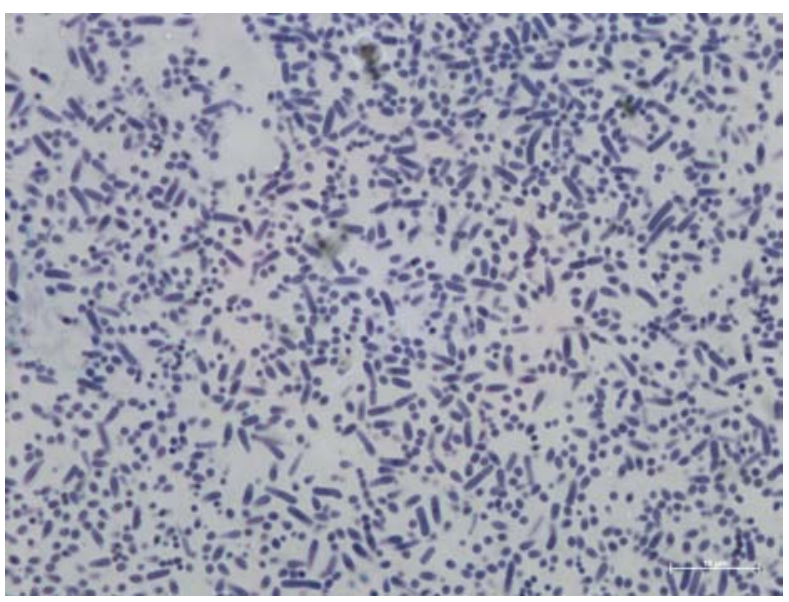

Figure 1. Light micrograph of M. mulieris. Bar $=10$ $\mu \mathrm{m}$.

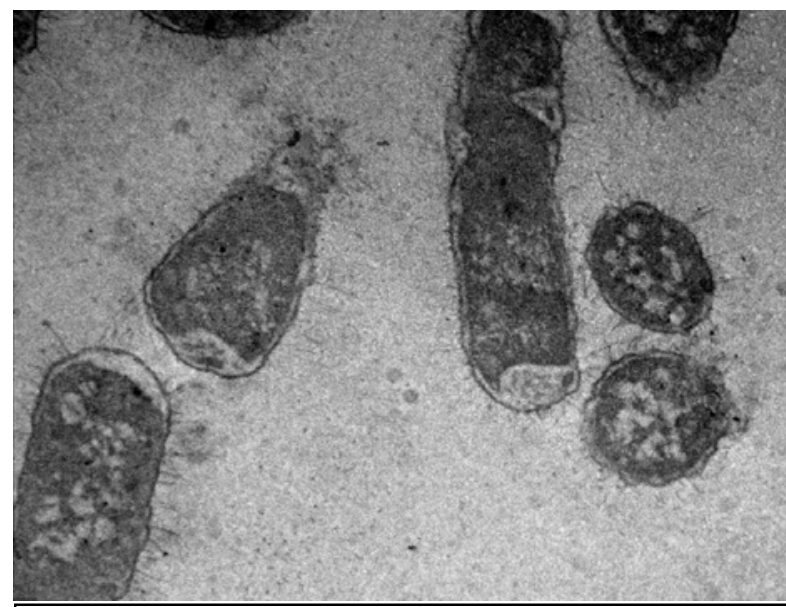

Figure 2. Transmission electron micrograph of $M$. mulieris showing cytoplasmic inclusions in some rods.

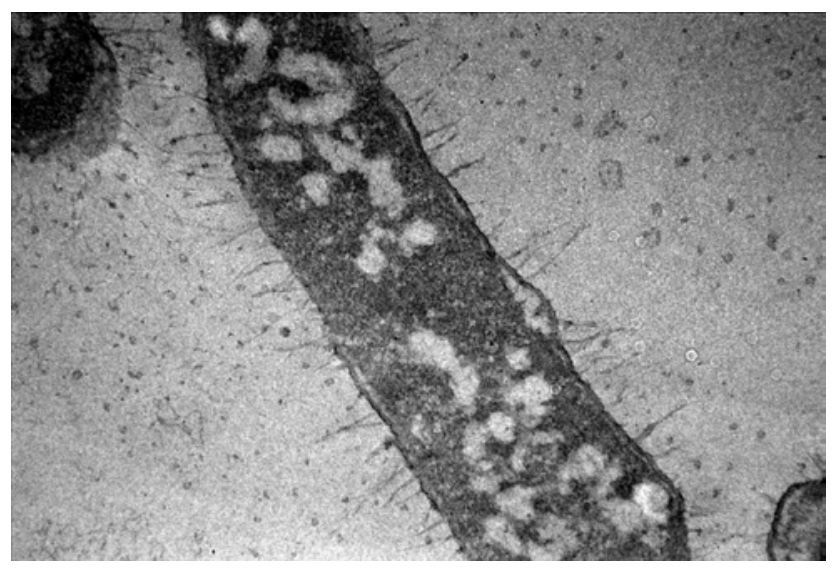

Figure 3. TEM of M. mulieris: numerous filamentous processes are observed emanating from the cell surface.

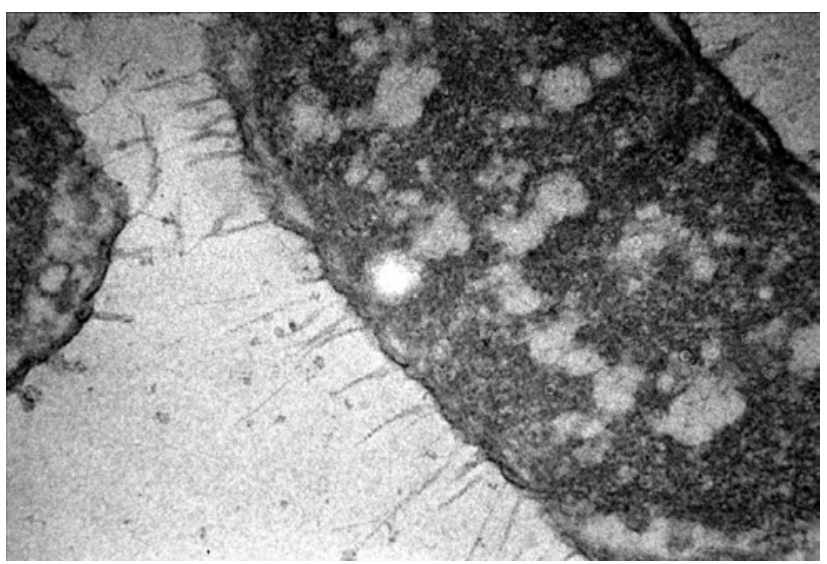

Figure 4. Higher resolution of the filaments, presumably pili.

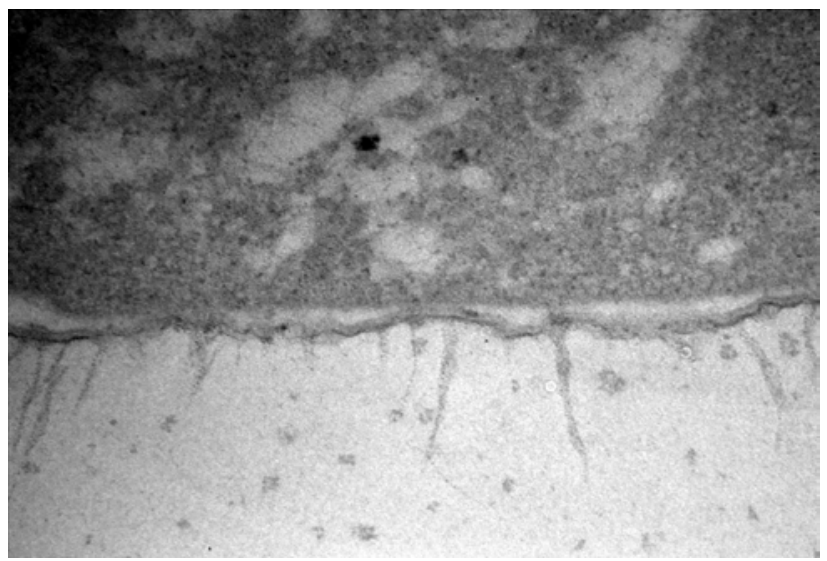

Figure 5. Higher resolution of the presumed pili. More work is being conducted on these structures. 